\title{
Majorana Single-Charge Transistor
}

\author{
R. Hützen, ${ }^{1}$ A. Zazunov, ${ }^{1}$ B. Braunecker, ${ }^{2}$ A. Levy Yeyati, ${ }^{2}$ and R. Egger $^{1}$ \\ ${ }^{1}$ Institut für Theoretische Physik, Heinrich-Heine-Universität, D-40225 Düsseldorf, Germany \\ ${ }^{2}$ Departamento de Física Teórica de la Materia Condensada C-V and Instituto Nicolás Cabrera, \\ Universidad Autónoma de Madrid, E-28049 Madrid, Spain
}

(Received 18 June 2012; published 16 October 2012)

\begin{abstract}
We study transport through a Coulomb blockaded topologically nontrivial superconducting wire (with Majorana end states) contacted by metallic leads. An exact formula for the current through this interacting Majorana single-charge transistor is derived in terms of wire spectral functions. A comprehensive picture follows from three different approaches. We find Coulomb oscillations with universal halving of the finitetemperature peak conductance under strong blockade conditions, where the valley conductance mainly comes from elastic cotunneling. The nonlinear conductance exhibits finite-voltage sidebands due to anomalous tunneling involving Cooper pair splitting.
\end{abstract}

DOI: 10.1103/PhysRevLett.109.166403

PACS numbers: 71.10.Pm, 73.23.-b, 74.50.+r

Introduction.-Topologically nontrivial insulators and superconductors exhibit many remarkable nonlocal features such as teleportation or non-Abelian statistics [1,2]. For a one-dimensional topological superconductor (TS) wire, such effects can be traced back to the existence of a zero-energy Majorana bound state (MBS) localized at each end [3-7]. When a grounded TS is weakly contacted by a normal metal, the MBS is expected to produce a characteristic zero-bias anomaly peak in the tunnel conductance [8-12]. Very recently, such a feature has been experimentally observed in tunnel spectroscopy using InSb or InAs nanowires [13-16], where Majorana fermions are theoretically expected due to the interplay of strong spin-orbit coupling, Zeeman field, and proximity-induced superconducting pairing [17-19]. Recent reviews [1,2,6,7,19] have also summarized alternative MBS proposals. Here we discuss an interacting variant of previously studied Majorana wire setups, the floating "Majorana single-charge transistor" (MSCT) schematically shown in Fig. 1. A comprehensive picture of its transport properties in the presence of interactions emerges from our analysis below. Noting that the experimentally observed peak features could be related to a disorder-induced spectral peak [20,21], our results should help to distinguish the Majorana state from alternative explanations in future experiments.

Previous works [22-24] have studied electron-electron interactions in an isolated TS wire and found that Majoranas still exist under rather general conditions. As sketched in Fig. 1, we instead study a generalization of the setup in Ref. [13], where source and drain metallic electrodes contact the TS wire. We stress that the MSCT could be realized not only with nanowires but using most other Majorana proposals as well. In such a geometry, Coulomb blockade effects due to the finite charging energy $E_{c}$ of the TS can play a decisive role. For instance, one expects Coulomb oscillations of the conductance as a function of a gate voltage parameter $n_{g}$, with peaks (valleys) near half-integer (integer) $n_{g}$, while in the noninteracting $\left(E_{c}=0\right)$ limit, the MBSs pinned to zero energy cause resonant Andreev reflection (AR) [8-11], with $n_{g}$-independent linear conductance $G=2 e^{2} / h$ at temperature $T=0$. Resonant $\mathrm{AR}$ also survives for $E_{c} \lesssim \Gamma=$ $\Gamma_{L}+\Gamma_{R}$, albeit with reduced conductance [25]. For $E_{c} \gg \Gamma$, the Coulomb blockade is firmly established, and the peak conductance approaches the (spinless) resonant tunneling value $G=e^{2} / h$, which has been pointed out as a signature of electron teleportation [26].

In this Letter, we consider Coulomb blockaded charge transport through the MSCT; for a variant with one superconducting and one metallic lead, see Ref. [27]. We provide an exact expression for the current in this interacting system, and develop three different approximation schemes to study Coulomb oscillations in the MSCT, both for $T=0$ and finite $T$. We quantitatively describe the $T=0$ crossover of the peak conductance from $G=$ $2 e^{2} / h$ to $e^{2} / h$ as $E_{c} / \Gamma$ increases, which constitutes a characteristic signature of Majoranas. Remarkably, this "halving" of the peak conductance is universal and found

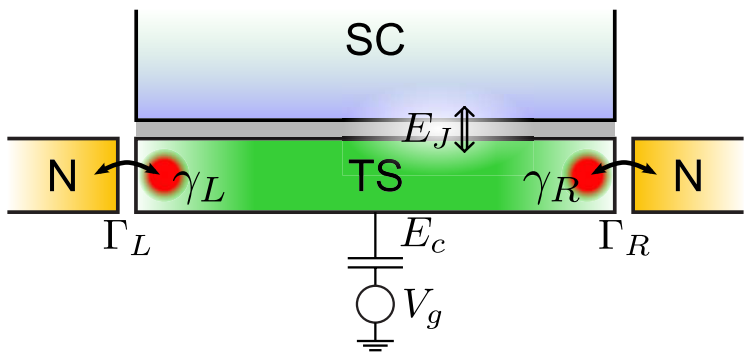

FIG. 1 (color online). Majorana single-charge transistor (MSCT): The TS wire with Majorana end states is tunnel coupled $\left(\Gamma_{L}, \Gamma_{R}\right)$ to normal metal electrodes and Josephson coupled $\left(E_{J}\right)$ to another bulk superconductor. Capacitive charging effects are encoded by $E_{c}$ and can be tuned by a gate voltage parameter $n_{g} \propto V_{g}$. 
to hold for arbitrary $T$. For the valley conductance, we find that elastic cotunneling dominates while AR is subleading. We predict finite-voltage sidebands in the nonlinear differential conductance which are directly related to anomalous tunneling processes where the Majorana state and the Cooper pair number change simultaneously. The presence of Majoranas can be unambiguously identified in experiments by the magnetic field dependence of the sideband location.

Model.-The MSCT Hamiltonian, $H=H_{c}+H_{t}+H_{l}$, contains a piece $H_{c}$ describing the TS wire, a tunnel Hamiltonian $H_{t}$ connecting the TS to the left $(j=L)$ and right $(j=R)$ electrode, and a term $H_{l}$ describing the leads (we often use units with $e=\hbar=k_{B}=1$ ). Topological arguments warrant that the TS wire holds a single unpaired MBS near each end [6,7] described by the Majorana operator $\gamma_{j}=\gamma_{j}^{\dagger}$ with anticommutator $\left\{\gamma_{j}, \gamma_{j^{\prime}}\right\}=\delta_{j j^{\prime}}$. We introduce the nonlocal fermion operator $d=\left(\gamma_{L}+i \gamma_{R}\right) / \sqrt{2}$, such that $\gamma_{L}=\left(d+d^{\dagger}\right) / \sqrt{2}$ and $\gamma_{R}=-i\left(d-d^{\dagger}\right) / \sqrt{2}$. With $\hat{n}_{d}=d^{\dagger} d$ and the number operator $\hat{N}$ for Cooper pairs in the TS, the instantaneous charge state of the wire is described by $\left(N, n_{d}\right)$, where the integer $N$ and $n_{d}=0,1$ are eigenvalues of $\hat{N}$ and $\hat{n}_{d}$, respectively. With the phase $\chi$ conjugate to $\hat{N}$, where $[\chi, \hat{N}]=i$ and $e^{-i \chi}\left(e^{i \chi}\right)$ lowers (raises) $N$ by one unit, we have

$$
H_{c}=E_{c}\left(2 \hat{N}+\hat{n}_{d}-n_{g}\right)^{2}-E_{J} \cos \left(\chi-\phi_{S}\right) .
$$

The TS wire is assumed sufficiently long to exclude a direct tunnel coupling between $\gamma_{L}$ and $\gamma_{R}$. However, note that $E_{c}$ introduces a dynamical coupling between the Majoranas. Proximity-induced pairing correlations are required for MBS formation, and in Eq. (1) we include Cooper pair exchange (with Josephson coupling $E_{J}$ ) between the TS condensate and another bulk superconductor (with fixed phase $\left.\phi_{S}\right)[27,28]$. We focus on the most interesting case of a large proximity gap $\Delta_{\mathrm{TS}}>\max \left(E_{c}, \Gamma, T\right)$, where charge transport involves MBSs and the contribution of quasi-particles above the gap can be neglected. Next, electrons in lead $j$ correspond to free fermions with chemical potential $\mu_{j}$ and (effectively spinless [25]) fermionic operators $c_{j, k}$ for momentum $k . H_{l}$ is treated within the usual wide-band approximation [29] and the bias voltage is $e V=\mu_{L}-\mu_{R}$. Taking into account charge conservation and expressing the Majoranas in terms of the nonlocal $d$ fermion, the tunnel Hamiltonian reads [25]

$$
H_{t}=\sum_{j} \lambda_{j} c_{j}^{\dagger} \eta_{j}+\text { H.c., } \quad \eta_{j}=\frac{1}{\sqrt{2}}\left(d+s_{j} e^{-i \chi} d^{\dagger}\right),
$$

where $c_{j}=\sum_{k} c_{j, k}, s_{L}=+1$ and $s_{R}=-1$, and $\lambda_{L, R}$ denotes the respective tunnel matrix elements [11]. Tunneling from the TS to lead $j$ thus proceeds either by destroying the $d$ state without changing $N$ ("normal" tunneling) or by occupying the $d$ state and simultaneously splitting a Cooper pair ("anomalous" tunneling), plus the conjugate processes. Below we use the hybridization scales $\Gamma_{j}=$ $2 \pi \nu_{j}\left|\lambda_{j}\right|^{2}$, where $\nu_{j}$ is the density of states in lead $j$. Experimentally, the $\Gamma_{j}$ (and $n_{g}$ ) can be changed via gate voltages [13].

Exact expression for current.-Using nonequilibrium Green's function (GF) techniques [30,31], the current $I_{j}$ flowing from lead $j$ to the TS can be expressed in terms of the Keldysh GF $\breve{G}_{\eta_{j}}\left(t, t^{\prime}\right)=-i\left\langle\mathcal{T}_{C} \eta_{j}(t) \eta_{j}\left(t^{\prime}\right)\right\rangle$, where $\widetilde{\mathcal{T}}_{C}$ denotes Keldysh time ordering and the pseudo-fermion $\eta_{j}$ has been defined in Eq. (2). With the Fourier-transformed retarded, $G_{\eta_{j}}^{R}(\epsilon)$, and Keldysh, $G_{\eta_{j}}^{K}(\epsilon)$, components of $\check{G}_{\eta_{j}}$, we obtain $I_{j}=\left(e \Gamma_{j} / h\right) \int d \epsilon\left[F\left(\epsilon-\mu_{j}\right) \operatorname{Im} G_{\eta_{j}}^{R}(\epsilon)+\right.$ $\left.(i / 2) G_{\eta_{j}}^{K}(\epsilon)\right]$, where $F(\epsilon)=1-2 f=\tanh (\epsilon / 2 T)$ encodes the Fermi function $f(\epsilon)$ in the leads. Next, we note that $G_{\eta_{j}}^{K}(t, t)=0$ as a consequence of $\eta_{j}^{\dagger} \eta_{j}=\eta_{j} \eta_{j}^{\dagger}=1 / 2$. Hence, we find the exact result

$$
I_{j}=\frac{e \Gamma_{j}}{h} \int d \epsilon F\left(\epsilon-\mu_{j}\right) \operatorname{Im} G_{\eta_{j}}^{R}(\epsilon),
$$

stating that the current can be computed from the spectral function $\propto \operatorname{Im} G_{\eta_{j}}^{R}$. The well-known expression for interacting quantum dots [32] has thereby been extended to the interacting Majorana wire; note that there are two spectral functions associated with the currents $I_{L}$ and $I_{R}$. Current conservation here implies $I_{L}+I_{R}+I_{S}=0$, with the supercurrent $I_{S}$ flowing through the interface to the bulk superconductor. Below we define the conductance $G=$ $d I / d V$ using the symmetrized current $I=\left(I_{L}-I_{R}\right) / 2$. For $E_{c}=0$, the pseudo-fermions $\eta_{j}$ reduce to Majorana fermions $\gamma_{j}$, and the Lorentzian spectral function, $-\operatorname{Im} G_{\gamma_{j}}^{R}(\epsilon)=\Gamma_{j} /\left(\epsilon^{2}+\Gamma_{j}^{2}\right)$, implies resonant AR with $G=2 e^{2} / h$ [8-10]. For finite $E_{c}$, we shall present several complementary approximations in order to achieve a broad physical understanding of the MSCT transport properties. Equation (3) should also be useful for numerically exact calculations, e.g., using the numerical or density-matrix renormalization group.

Equation-of-motion (EOM) approach.-We constructed an EOM approach for $G_{\eta_{j}}^{R}$ to access the linear conductance near a peak. Within this method, we introduce the Nambu spinors $\Psi_{d}=\left(d, e^{-i \chi} d^{\dagger}\right)^{T}$ and the corresponding retarded $\mathrm{GF}, G_{d d}^{R}=-i \Theta\left(t-t^{\prime}\right)\left\langle\left\{\Psi_{d}(t), \Psi_{d}^{\dagger}\left(t^{\prime}\right)\right\}\right\rangle$. The EOM for $G_{d d}^{R}$ generates higher-order GFs of the type $\Gamma_{N^{m} d d}^{R}=$ $-i \Theta\left(t-t^{\prime}\right)\left\langle\left\{\hat{N}^{m}(t) \Psi_{d}(t), \Psi_{d}^{\dagger}\left(t^{\prime}\right)\right\}\right\rangle$, which we truncate at the level $m=2$ and solve in a self-consistent way [33]. The resulting GF $G_{d d}^{R}$ then yields $G_{\eta_{j}}^{R}=\frac{1}{2} \operatorname{Tr}[(1+$ $\left.s_{j} \sigma_{x}\right) G_{d d}^{R}$ ] with Pauli matrix $\sigma_{x}$. Finally, we obtain the conductance from Eq. (3). This approximation is valid by construction for $E_{c} \gtrsim \Gamma$, but the imposed self-consistency [33] allows us to extend it to $E_{c}<\Gamma$, where the resulting conductance (being determined by truncated fluctuations) gives a lower bound for the exact result. 
Zero-bandwidth model (ZBWM).-Next we study the ZBWM where each lead is represented by just a single fermion site and only a finite number of TS Cooper pairs $\left(N<N_{\max }\right)$ is included. The Hilbert space then has the finite dimension $8 N_{\max }$, which allows us to numerically calculate the spectral density $\propto \operatorname{Im} G_{\eta_{j}}^{R}(\epsilon)$ via its Lehmann representation, with poles phenomenologically broadened by $\Gamma$. A similar description has been pursued before for $E_{c}=E_{J}=0$ [34]. With this spectral function, Eq. (3) yields the conductance within the ZBWM.

Master equation and cotunneling processes.-For $T>\Gamma$, the GF formulation reduces to a master equation description including sequential tunneling and cotunneling processes (for simplicity, $E_{J}=0$ here). The stationary probability distribution $P_{Q}$ for having $Q=2 N+n_{d}$ particles on the TS then obeys $\sum_{Q^{\prime} \neq Q}\left[P_{Q^{\prime}} W_{Q^{\prime} \rightarrow Q}-P_{Q} W_{Q \rightarrow Q^{\prime}}\right]=0$. All nonvanishing transition rates $W_{Q \rightarrow Q^{\prime}}$ are specified in terms of rates obtained under a systematic second-order $T$-matrix expansion in $\Gamma_{L, R}$ [35]. With the electrostatic energy $E_{Q}=E_{c}\left(Q-n_{g}\right)^{2}$, sequential tunneling yields the rate $\Gamma_{j, Q \rightarrow Q \pm 1}^{(\mathrm{seq})}=\left(\Gamma_{j} / 2\right) f\left(E_{Q \pm 1}-E_{Q} \mp \mu_{j}\right)$ for one particle tunneling into (out of) the TS from (to) lead
$j=L, R$. Next, elastic cotunneling transfers a particle from lead $j$ to the opposite lead $-j$ with virtual excitation of the TS states $Q \pm 1$. The elastic cotunneling rate is

$$
\begin{aligned}
\Gamma_{j, Q}^{(\mathrm{EC})}= & \frac{\Gamma_{L} \Gamma_{R}}{8 \pi} \int d \epsilon f\left(\epsilon-\mu_{j}\right)\left[1-f\left(\epsilon-\mu_{-j}\right)\right] \\
& \times \mid \frac{1}{\epsilon-\left(E_{Q+1}-E_{Q}\right)+i 0} \\
& -\left.\frac{1}{\epsilon-\left(E_{Q}-E_{Q-1}\right)-i 0}\right|^{2}
\end{aligned}
$$

where the two terms come from the interference of normal and anomalous tunneling. We note in passing that for large $\Delta_{\text {TS }}$, inelastic cotunneling does not contribute at all, while the conventional elastic cotunneling rate due to quasiparticle states above the gap (and without MBSs) would be much smaller, $\Gamma^{(\mathrm{EC})} \propto \Gamma_{L} \Gamma_{R} / \Delta_{\mathrm{TS}}$ [30]. To the same order in $\Gamma_{L, R}$, we also have local (and crossed) AR processes, where an electron and a hole from the same (different) lead(s) are combined to form a Cooper pair, $Q \rightarrow Q+2$; the reverse process describes Cooper pair splitting, $Q \rightarrow Q-2$. Some algebra yields the AR rates

$$
\begin{aligned}
\Gamma_{j, j^{\prime}, Q \rightarrow Q \pm 2}^{(\mathrm{AR})}= & \frac{1+\delta_{j,-j^{\prime}}}{2} \frac{\Gamma_{j} \Gamma_{j^{\prime}}}{8 \pi} \int d \epsilon \int d \epsilon^{\prime} f\left( \pm\left(\epsilon-\mu_{j}\right)\right) f\left( \pm\left(\epsilon^{\prime}-\mu_{j^{\prime}}\right)\right) \delta\left(\epsilon+\epsilon^{\prime} \mp\left(E_{Q \pm 2}-E_{Q}\right)\right) \\
& \times\left|\frac{1}{\epsilon \mp\left(E_{Q \pm 1}-E_{Q}\right)+i 0}-\frac{s_{j} s_{j^{\prime}}}{\epsilon^{\prime} \mp\left(E_{Q \pm 1}-E_{Q}\right)+i 0}\right|^{2},
\end{aligned}
$$

where $j=j^{\prime}\left(j \neq j^{\prime}\right)$ corresponds to local (crossed) AR. The $i 0$ terms indicate that regularization of the integrals in Eqs. (4) and (5) is necessary. Application of the general regularization scheme in Refs. $[36,37]$ then implies that the principal value of these integrals needs to be taken. Given these rates and the (numerical) solution $P_{Q}$ of the master equation, the currents $I_{j}$ then readily follow.

Coulomb oscillations. - Let us first address the $n_{g}$ dependence of the linear $(V \rightarrow 0)$ conductance, see Fig. 2; we take $\Gamma_{L}=\Gamma_{R}=\Gamma / 2$ in all figures. Both the master equation (main panel, finite $T$ ) and the ZBWM (inset, $T=0$ ) reveal clear conductance oscillations in the MSCT for $E_{c} \gg \Gamma$, with peaks (valleys) for half-integer (integer) gate voltage parameter $n_{g}$. The main panel shows that the peak (valley) conductance is halved (strongly suppressed) when going from the noninteracting to the deep Coulomb blockade limit. For $E_{J}=0$ and $(\Gamma, T) \ll E_{c}$, the line shape of the valley conductance is obtained in closed form,

$$
G_{\text {valley }}(\delta)=\frac{e^{2}}{h} \frac{\Gamma_{L} \Gamma_{R}}{E_{c}^{2}} \frac{1}{\left(1-4 \delta^{2}\right)^{2}},
$$

where $\delta=n_{g}-\left[n_{g}\right]$ with $|\delta| \ll 1$ is the deviation from a valley center. Equation (6) comes from elastic cotunneling, with constructive interference of the normal and anomalous tunneling contributions [see Eq. (4)], while AR is strongly suppressed in this limit. The inset of Fig. 2 shows that $G$ increases when the Josephson coupling $E_{J}$ grows. One can understand this by noting that for $E_{J} \gg E_{c}$, one ultimately reaches the resonant AR limit of a grounded TS, with the $n_{g}$-independent $T=0$ conductance $G=2 e^{2} / h$.

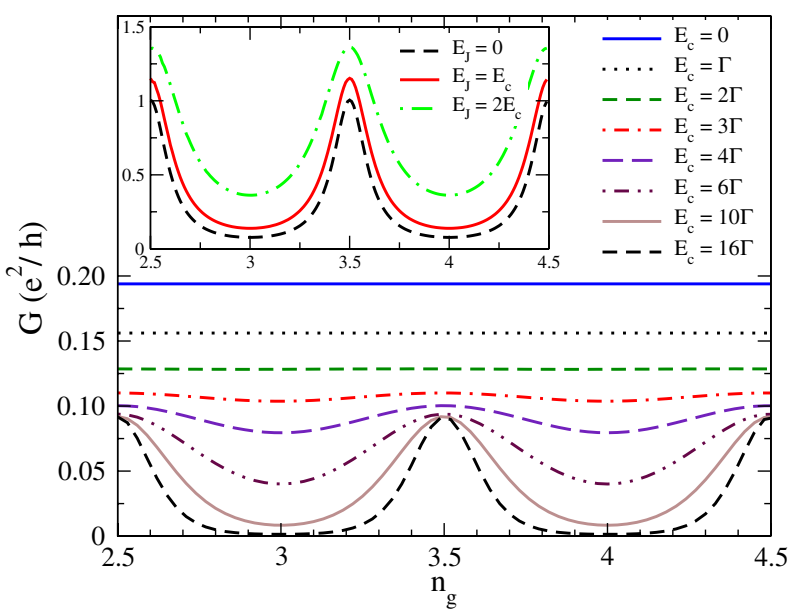

FIG. 2 (color online). Coulomb oscillations in the MSCT. Main panel: Conductance $G$ vs $n_{g}$ from the master equation for $E_{J}=0, T=2 \Gamma$ and several $E_{c}$. Inset: Same but from ZBWM for $T=0, E_{c}=5 \Gamma$, and several $E_{J}$. 


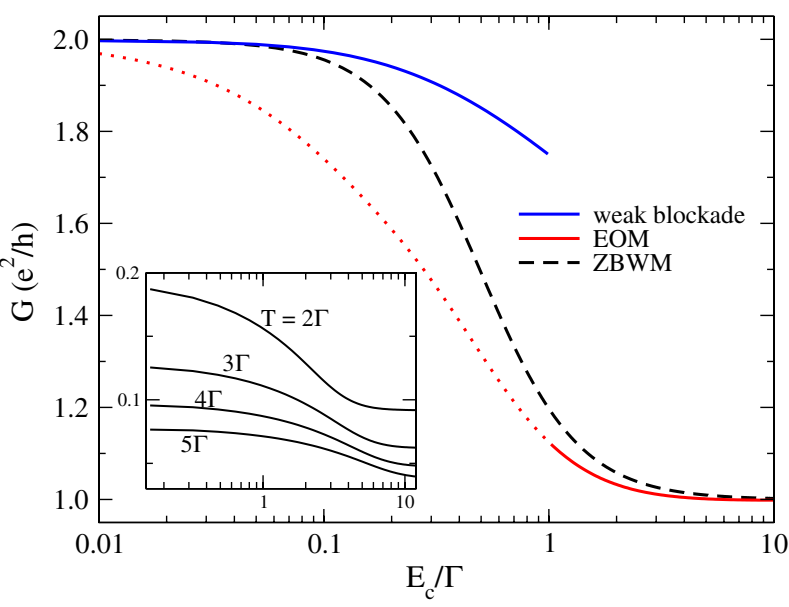

FIG. 3 (color online). Peak conductance $G$ vs $E_{c} / \Gamma$ on a semilogarithmic scale. Main panel: Comparison of $T=0$ results using perturbation theory in $E_{c} / \Gamma$ [25] (blue solid curve), the EOM approach (red dotted-solid curve), and the ZBWM (black dashed curve). The shown EOM results are quantitatively valid only for $E_{c} \gtrsim \Gamma$ (solid part) but give a lower bound when $E_{c} \lesssim \Gamma$ (dotted part). Here, $E_{J}=0$ since $G$ only weakly depends on $E_{J}$ for $E_{J} \lesssim E_{c}$. Inset: Same but from the master equation for several temperatures $T>\Gamma$.

We find that AR yields significant conductance contributions for $E_{J} \gtrsim E_{c}$, which are best detected through the nonlocal conductance $\partial I_{L} / \partial \mu_{R}$. However, we will discuss this quantity in detail elsewhere.

Peak conductance.-Results for the peak conductance are shown in Fig. 3. For $T=0$ (main panel), we obtain the full crossover from $G=2 e^{2} / h$ to $G=e^{2} / h$ as $E_{c} / \Gamma$ increases. The known small- $E_{c}$ behavior [25] is nicely reproduced by the ZBWM calculation. In the opposite large- $E_{c}$ limit, the EOM method is very accurate and Fig. 3 suggests that the simple ZBWM already captures the crossover from resonant AR [8-10] to electron teleportation [26] surprisingly well. The inset of Fig. 3 again demonstrates the universal halving of the finite- $T$ peak conductance; see also Fig. 2. Since experiments so far were conducted in the high-temperature regime $T>\Gamma$ [13], let us now specify the line shape near a conductance peak for $E_{c} \gg \Gamma$. Using $\delta=n_{g}-\left[n_{g}\right]-1 / 2$ with $|\delta| \ll 1$ for the deviation from a peak center, truncation of the master equation to two charge states gives

$$
G_{\mathrm{peak}}(\delta)=\frac{e^{2}}{h} \frac{\pi \Gamma}{16 T} \frac{1}{\cosh ^{2}\left(\delta E_{c} / T\right)} .
$$

We stress that the peak conductance $\left[G_{\text {peak }}(\delta=0)\right]$ is indeed halved compared to $E_{c}=0$ [8]. Moreover, it exhibits both a thermal and an interaction-induced reduction.

Finite-voltage sidebands. - Next we discuss the differential conductance at finite bias voltage $V$. Master equation results for $T=2 \Gamma$ are shown in Fig. 4. Starting with the main panel, we find sideband peaks when $\mathrm{eV}$ is equal to an

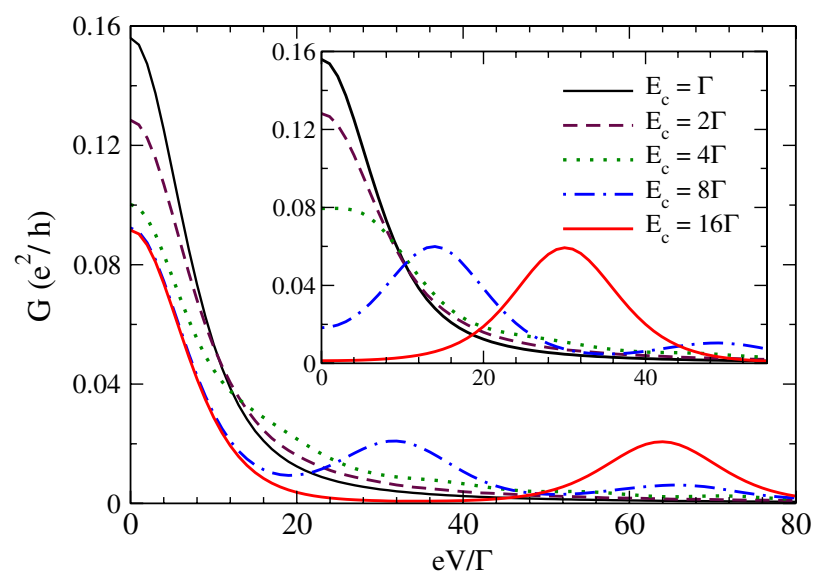

FIG. 4 (color online). $G=d I / d V$ vs voltage $V$ from the master equation for $T=2 \Gamma, E_{J}=0$, and several $E_{c} / \Gamma$. The main panel (inset) is for half-integer (integer) $n_{g}$.

integer multiple of $4 E_{c}$. For these voltages, the chemical potentials $\mu_{L, R}$ are resonant with two (almost) degenerate higher-order charge states, implying additional sequential tunneling contributions beyond the resonant transition determining the linear conductance peak [Eq. (7)]. Note that the fluctuations in $N$ needed to reach higher-order charge states can only be achieved through anomalous tunneling processes [see Eq. (2)]. Similar sideband peaks are also found for other $n_{g}$; the integer- $n_{g}$ valley case is shown in the inset of Fig. 4. In Fig. 5 we show the evolution of the sideband peaks as $E_{J}$ is changed, determined from the ZBWM at $T=0$. For half-integer $n_{g}$, the sideband peak position observed in the main panel of Fig. 5 is well described by $e V \simeq 4 E_{c} \sqrt{1+\left(E_{J} / 2 E_{c}\right)^{2}}$, which comes from Josephson coupling between the two relevant charge states. Since $E_{J}$ can be tuned by applying a small magnetic field parallel to the junction between the TS and the bulk superconductor, an experimental observation of the sideband peak and its shift with magnetic field

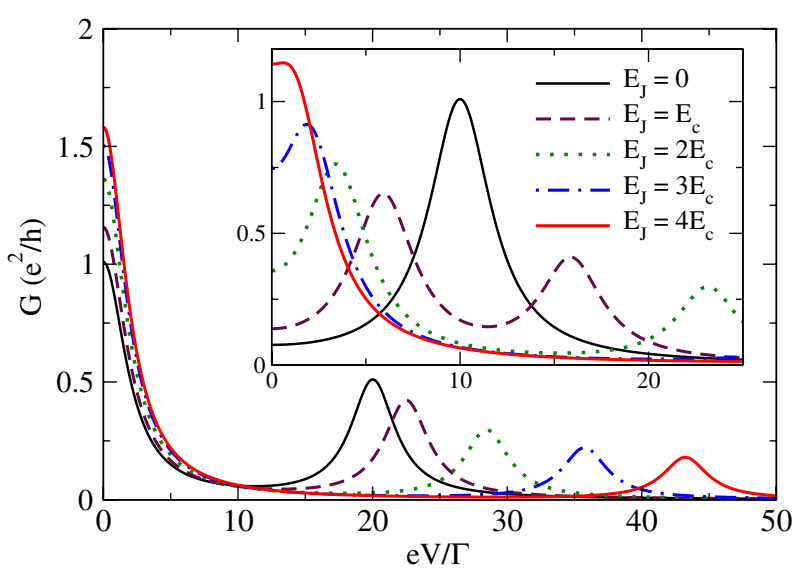

FIG. 5 (color online). Same as Fig. 4 but from ZBWM for $T=0, E_{c}=5 \Gamma$, and several $E_{J}$. 
[cf. the expression for the peak position above] would provide clear evidence for the anomalous tunneling mechanism, and thereby for the presence of MBSs.

Conclusions. - In this Letter, we have studied the transport properties of an interacting Majorana single-charge transistor. Our results should be directly relevant for experiments extending existing work, see, e.g., Ref. [13], where conductance peaks for tunneling into Majorana wires were reported. When a gate voltage parameter $n_{g}$ is varied, we find Coulomb oscillations, where the behavior of the peak and valley conductance has been characterized in detail. The Majorana fermions in this system could be identified by observing sideband peaks in the nonlinear conductance and from the crossover behavior of the Coulomb peak conductance.

This work was supported by the DFG (Grant No. EG-96/ 9-1 and SFB TR 12), by the EU network SE2ND, and by the Spanish MICINN under Contract FIS2008-04209.

Note added.-After submission of this work, we learned of an unpublished study of the MSCT model by $\mathrm{Fu}$ and Kane [38].

[1] M. Z. Hasan and C. L. Kane, Rev. Mod. Phys. 82, 3045 (2010).

[2] X. L. Qi and S.C. Zhang, Rev. Mod. Phys. 83, 1057 (2011).

[3] A. Yu. Kitaev, Phys. Usp. 44, 131 (2001).

[4] L. Fu and C. L. Kane, Phys. Rev. Lett. 100, 096407 (2008).

[5] M. Sato and S. Fujimoto, Phys. Rev. B 79, 094504 (2009).

[6] C. W. J. Beenakker, arXiv:1112.1950.

[7] M. Leijnse and K. Flensberg, arXiv:1206.1736.

[8] C. J. Bolech and E. Demler, Phys. Rev. Lett. 98, 237002 (2007).

[9] J. Nilsson, A. R. Akhmerov, and C. W. J. Beenakker, Phys. Rev. Lett. 101, 120403 (2008).

[10] K. T. Law, P. A. Lee, and T. K. Ng, Phys. Rev. Lett. 103, 237001 (2009).

[11] K. Flensberg, Phys. Rev. B 82, 180516(R) (2010).

[12] M. Wimmer, A. R. Akhmerov, J. P. Dahlhaus, and C. W. J. Beenakker, New J. Phys. 13, 053016 (2011).

[13] V. Mourik, K. Zuo, S. M. Frolov, S. R. Plissard, E. P. A. M. Bakkers, and L. P. Kouwenhoven, Science 336, 1003 (2012).

[14] L. Rokhinson, X. Liu, and J. Furdyna, arXiv:1204.4212.

[15] A. Das, Y. Ronen, Y. Most, Y. Oreg, M. Heiblum, and H. Shtrikman, arXiv:1205.7073.
[16] M. T. Deng, C. L. Yu, G. Y. Huang, M. Larsson, P. Caroff, and H. Q. Xu, arXiv:1204.4130.

[17] R. M. Lutchyn, J.D. Sau, and S. Das Sarma, Phys. Rev. Lett. 105, 077001 (2010)

[18] Y. Oreg, G. Refael, and F. von Oppen, Phys. Rev. Lett. 105, 177002 (2010).

[19] J. Alicea, Rep. Prog. Phys. 75, 076501 (2012).

[20] D. Bagrets and A. Altland, arXiv:1206.0434.

[21] J. Liu, A.C. Potter, K. T. Law, and P. A. Lee, arXiv:1206.1276.

[22] S. Gangadharaiah, B. Braunecker, P. Simon, and D. Loss, Phys. Rev. Lett. 107, 036801 (2011).

[23] E. M. Stoudenmire, J. Alicea, O. A. Starykh, and M. P. A. Fisher, Phys. Rev. B 84, 014503 (2011).

[24] E. Sela, A. Altland, and A. Rosch, Phys. Rev. B 84, 085114 (2011).

[25] A. Zazunov, A. L. Yeyati, and R. Egger, Phys. Rev. B 84, 165440 (2011).

[26] L. Fu, Phys. Rev. Lett. 104, 056402 (2010).

[27] N. Didier, M. Gibertini, A. G. Moghaddam, J. König, and R. Fazio, arXiv:1202.6357.

[28] A. Zazunov and R. Egger, Phys. Rev. B 85, 104514 (2012).

[29] It is straightforward to go beyond this approximation by allowing for energy-dependent $\Gamma_{j}(\epsilon)$ in the equations below.

[30] Yu. V. Nazarov and Ya. M. Blanter, Quantum Transport (Cambridge University Press, Cambridge, England, 2009).

[31] A. Altland and B. Simons, Condensed Matter Field Theory (Cambridge University Press, Cambridge, England, 2010), 2nd ed.; G. Dolcetto, S. Barbarino, D. Ferraro, N. Magnoli, and M. Sassetti, Phys. Rev. B 85, 195138 (2012); M. Grifoni, M. Sassetti, and U. Weiss, Phys. Rev. E 53, R2033 (1996).

[32] Y. Meir and N. S. Wingreen, Phys. Rev. Lett. 68, 2512 (1992).

[33] See Supplemental Material at http://link.aps.org/ supplemental/10.1103/PhysRevLett.109.166403 for a brief summary of our EOM approach.

[34] S. Tewari, C. Zhang, S. Das Sarma, C. Nayak, and D. H. Lee, Phys. Rev. Lett. 100, 027001 (2008).

[35] We find $W_{Q \rightarrow Q \pm 1}=\sum_{j} \Gamma_{j, Q \rightarrow Q \pm 1}^{(\mathrm{seq})}$, and $W_{Q \rightarrow Q \pm 2}=$ $\Gamma_{L R, O \rightarrow Q \pm 2}^{(\mathrm{AR})}+\sum_{j} \Gamma_{j j, O \rightarrow Q \pm 2}^{(\mathrm{AR})}$. Additional rates (not specified here) involving Cooper pair transfer between the TS and the bulk superconductor are included for finite $E_{J}$.

[36] M. Turek and K. A. Matveev, Phys. Rev. B 65, 115332 (2002).

[37] J. Koch, F. von Oppen, Y. Oreg, and E. Sela, Phys. Rev. B 70, 195107 (2004).

[38] L. Fu and C. L. Kane (unpublished). 